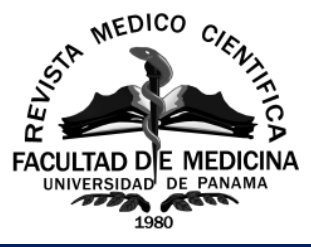

\title{
SÍNDROME PULMONAR POR HANTAVIRUS: UN ARTÍCULO DE REVISIÓN
}

\author{
HANTAVIRUS PULMONARY SYNDROME: A REVIEW ARTICLE \\ Raúl González-Castillo®*, Lorena Vanessa Alvarado®†, Tania J. Gallardo® \\ * Médico General, Universidad Latina de Panamá, Ciudad de Panamá, Panamá \\ †Médico General, Universidad de Panamá, Ciudad de Panamá, Panamá
}

\section{RESUMEN}

ANTECEDENTES: El síndrome pulmonar por hantavirus es ocasionado por el virus del orden Bunyavirales, familia Hantaviridae. Descrito por primera vez en un brote de casos en Estados Unidos en 1993 y actualmente con más de quince serotipos en América. Tiene una alta mortalidad y prevalencia variable; transmitido por roedores que, por los cambios climáticos y los movimientos migratorios, tienen más contacto con el ser humano. Presenta tropismo endotelial por el sistema respiratorio, con hallazgos de infiltrados pulmonares $y$ trombocitopenia, desarrollando en pocos días un edema pulmonar no cardiogénico y choque. No se cuenta actualmente con tratamiento específico ni vacuna.

OBJETIVO: Exponer el origen del síndrome pulmonar, comprender su epidemiología, fisiopatología, manifestaciones, investigar sobre los métodos diagnósticos y posibles tratamientos.

DISEÑO: Utilizamos los motores de búsqueda Pubmed, Cochrane, Science Direct y Scielo; delimitando artículos científicos publicados en inglés y español. Recopilando 148 publicaciones entre reporte de casos, estudios observacionales y experimentales, la mayoría entre los años 2010 y 2020.

CONCLUSIÓN: Las infecciones por hantavirus son diversas, donde progresivamente hemos podido entender su evolución, reservorios, formas de contagio, factores de riesgo, distribución mundial y las principales áreas endémicas para desarrollar estrategias de salud pública. No solo es una enfermedad zoonótica, sino también una enfermedad emergente que requiere atención multidisciplinaria para el implemento de nuevas terapias.

\section{ABSTRACT}

BACKGROUND: Hantavirus pulmonary syndrome is caused by a virus from the order Bunyavirales, family Hantaviridae. First described in 1993, during a case outbreak in the United States. Nowadays, it has more than fifteen serotypes in America, with high mortality and variable prevalence. Infected rodents carry the virus. Rodents are animals in close contact with humans because of climate changes and migration patterns. The viral endothelial tropism affects the respiratory system, producing as a consequence pulmonary infiltrate as well as thrombocytopenia, with progression to noncardiogenic pulmonary edema and shock in a matter of a few days. There is no specific treatment or vaccine for hantavirus infection.

OBJECTIVES: Present the Hantavirus Pulmonary Syndrome origin; understand its epidemiology, physiopathology, and clinical manifestations; acknowledge diagnostic methods and possible treatments.

DESIGN: We used medical search engines such as Pubmed, Cochrane, Science Direct and Scielo. We included only published scientific articles in English and Spanish. We collected 148 publications between 2010 and 2020, such as case reports, observational and experimental studies.

CONCLUSION: Hantavirus infections are diverse in terms of evolution, reservoirs, ways of transmission, risk factors, global distribution and principal endemic areas. Understanding those aspects allows to elaborate public health strategies. It is not only a zoonotic disease, but an emergent disease that demands multidisciplinary management for the indication of new therapies.

\begin{abstract}
Correspondencia: raulhgonzalezc@gmail.com Recibido: 27/02/21 Aceptado: 17/08/21
\end{abstract}

\section{PALABRAS CLAVE:}

Sindrome pulmonar por hantavirus, infecciones por hantavirus, hantavirus, zoonosis.

KEY WORDS:

Hantavirus pulmonary syndrome, hantavirus infections, hantavirus, zoonoses.
Las enfermedades por hantavirus (HV) son ocasionadas por variantes del orden Bunyavirales, familia Hantaviridae. ${ }^{[1]}$ Estos virus presentan morfología esférica, partículas de viriones, diámetro de 90-110 nm y material genético de ácido ribonucleico (ARN). Se encuentran compuestos de tres nucleocápsides helicoidales envueltos en una capa lipídica, dos glicoproteínas están asociadas con la envoltura, un nucleopéptido forma la estructura de la nucleocápside y un genoma con tres segmentos de oligonucleótidos pequeño (S), mediano (M), largo (L) con secuencias terminales 3' AUCAUCAUCUG (Ver Figura 1). ${ }^{[2]}$ 
La familia Hantaviridae tiene a los roedores como principal transmisor. Las otras familias pertenecientes al orden son Arenaviridae, Cruliviridae, Fimoviridae, Mypoviridae, Nairoviridae, Peribunyaviridae, Phasmaviridae, Phenuiviridae y Wupedeviridae. ${ }^{[1]}$

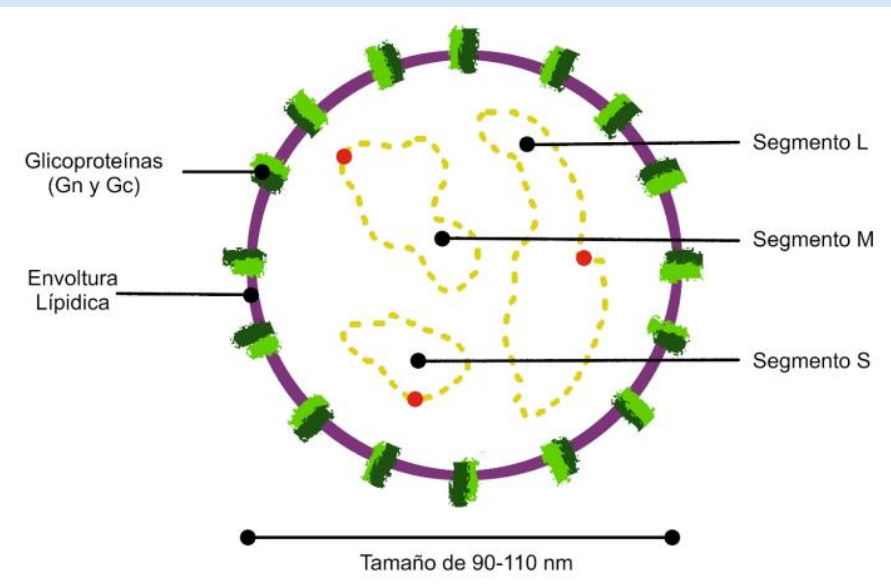

Figura 1. Estructura molecular del hantavirus

Fuente: Elaboración propia. Diseñada por Raúl GonzálezCastillo.

Examinando la información existente y el desconocimiento de esta patología importante en el continente americano, consideramos oportuno el aprendizaje sobre esta enfermedad infecciosa, particularmente, sobre el cuadro clínico que se manifiesta en la región. Por consiguiente, expondremos el origen de este síndrome, su epidemiología, fisiopatología, manifestaciones, diagnósticos y tratamiento.

\section{ANTECEDENTES}

Los virus del nuevo mundo causan síndrome pulmonar por hantavirus (SPH), en cambio los del viejo mundo ocasionan el síndrome renal hemorrágico por hantavirus (SRHH). A finales de la década de 1970 se aisló el virus Hantaan (HTNV) en el roedor Apodemus agrarius. Su historia se remonta a la Guerra de Corea, donde la enfermedad que presentaron los soldados se conoció como fiebre hemorrágica coreana; esta agrupaba los síntomas de fiebre, escalofríos, cefalea, mialgias, dolor abdominal y manifestaciones hemorrágicas. Posteriormente, se comienzan a aislar nuevos serotipos: virus Puumala (PUUV) en Finlandia, virus Hokkaido en Japón, virus Muju y virus Seoul en Corea del Sur. ${ }^{[3]}$ Un descubrimiento relativamente reciente aunque existen textos que describen síntomas similares al SRHH desde 960 d. C. ${ }^{[4]}$

El SPH se define por primera vez en 1993 durante el brote del suroeste de Estados Unidos, en donde se aísla el virus Sin Nombre del roedor Promiscuas maniculatus, el cual se encontraba en el peridomicilio de los pacientes. Las manifestaciones clínicas fueron insuficiencia respiratoria e infiltrado intersticial pulmonar bilateral sin síntomas prodrómicos. Aunado a esto, algunos casos desarrollaron depresión del miocardio en etapa crítica de la enfermedad. ${ }^{[4]}$ Años después en Latinoamérica se fueron descubriendo nuevos serotipos como virus Oran y Maciel en Argentina, virus Andes en Argentina y Chile, virus Juquitiba en Brasil, virus Choclo y Calabazo en Panamá. Más de 15 serotipos, todos responsables de esta enfermedad emergente. ${ }^{[5]}$

\section{EPIDEMIOLOGÍA}

EI SPH abarca desde un cuadro febril leve hasta llegar a complicaciones graves como choque cardiogénico e insuficiencia respiratoria de rápida instauración. Presenta un periodo de incubación de 4-42 días. A pesar de su prevalencia variable tiene mucha relevancia por su alta letalidad. ${ }^{[6]}$ Se sospechaba que la prevalencia variable en el continente americano era causada por la teoría del efecto de dilución, fenómeno para enfermedades infecciosas donde la diversidad de especies y cantidad de reservorios puede influir en su prevalencia. Sin embargo, aún no 
se ha comprobado que existe relación entre la teoría del efecto de dilución y la prevalencia en el nuevo mundo. ${ }^{[7]}$ Nuevos reportes de casos de SPH demuestran su presencia fuera de las Américas. En 2016, Alemania describe un caso por PUUV que evolucionó con síndrome de insuficiencia respiratoria aguda, progresó a falla circulatoria y requirió oxigenación por membrana extracorpórea, siendo estas manifestaciones del SPH. ${ }^{[8]}$

La transmisión al humano ocurre por inhalación de aerosoles, virus cargados en orina, salina o excretas del roedor (Ver Figura 2). ${ }^{[3]}$ Hay indicios de otras posibles formas de transmisión como mordedura del roedor y de persona a persona para el virus Andes. ${ }^{\text {[9] }}$ El movimiento migratorio humano y ocupaciones de áreas no habitadas propicia un aumento de casos. Vectores ambientales afectados por los cambios climáticos como el aumento de la temperatura (aumenta la sobrevida del roedor), menor cantidad de precipitaciones (disminuye la disponibilidad de alimentos lo que ocasiona que el roedor entre a las casas) y precipitaciones en exceso (disponibilidad excesiva de alimentos y mayor población de roedores) también ocasiona un aumento de casos. ${ }^{[10,11]}$

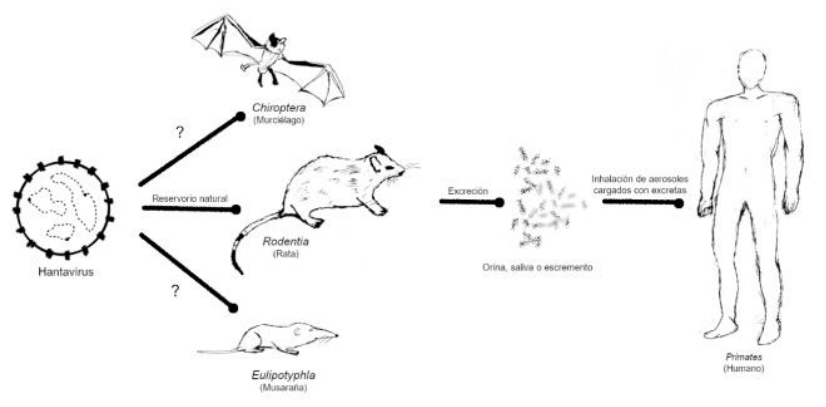

Figura 2. Cadena de transmisión del hantavirus en el ser humano.

Fuente: Elaboración propia. Diseñada por Raúl GonzálezCastillo.
La coevolución entre el virus y el huésped remonta a millones de años. Los árboles filogenéticos tanto de los virus como los reservorios presentan patrones similares. Se evidencia que el virus y su roedor en presencia de factores biogeográficos (migraciones, aislamiento del roedor, separación geográfica) son responsables de la distribución mundial actual. ${ }^{[12]}$ Recientes investigaciones en Europa Central han llevado al descubrimiento de serotipos importantes con más de un reservorio, lo que lleva a la comunidad científica a seguir buscando seroprevalencia en otros animales, y nuevas regiones endémicas posibles. ${ }^{[13]}$ Su principal reservorio son roedores del orden Rodentia, familia Muridae para el SRHH y subfamilia Sigmodontinae para el $\mathrm{SPH} .{ }^{[5]}$ Otros reservorios descritos han sido las musarañas o murciélagos. ${ }^{[3]}$

Los factores de riesgo para desarrollar SPH son: personas que viven o trabajan en áreas endémicas, áreas forestales y/o con espacios mal ventilados (graneros y almacenes de madera), sexo masculino, oficios con contacto animal, agricultura, militares. Las mujeres presentan mayor mortalidad y severidad de la enfermedad que los hombres, pero no se encontró significancia estadística con respecto a la edad. ${ }^{[3]}$ El virus puede ser eliminado con limpieza frecuente de las áreas con sustancias como: detergentes, solventes orgánicos o soluciones con hipoclorito, recolección de la basura y evitar la presencia de roedores en el peridomicilio. ${ }^{[14]}$

FISIOPATOLOGÍA

Un modelo animal actual para extrapolar datos sobre infecciones por HV es limitado. A pesar de que se ha intentado reproducir sin éxito el $\mathrm{SPH}$ en ciertos roedores, no es hasta en el año 2017 que Vergote et al. demostró signos clínicos de SPH, incluido edema pulmonar, en hámsteres sirios dorados inmunocomprometidos con la inoculación del virus Sin Nombre y otras especies de HV del nuevo mundo

www.revistamedicocientifica.org 
y así permitiendo entender más como se desarrolla el SPH. ${ }^{[15]}$

El mecanismo de entrada del virus está parcialmente comprendido y se sugiere que es un proceso dependiente de receptores de integrina 81 o 83 , abundantes en la superficie de plaquetas y células endoteliales. ${ }^{[16]}$ Posterior a su entrada y transcripción del material genético viral, su maduración ocurre en el aparato de Golgi y la superficie celular; el virus se disemina por células dendríticas inmaduras que se localizan cerca del epitelio celular de las vías respiratorias y viajando con viriones a través de los vasos linfáticos hacia los nódulos linfáticos. En el ser humano el SPH tiene tropismo principalmente por el tejido endotelial del sistema respiratorio $y$, en menor medida, el sistema cardiovascular, hepático y renal. ${ }^{[17]} \mathrm{Su}$ patogénesis radica fundamentalmente en el aumento de la permeabilidad vascular y trombocitopenia; además, la unión con integrinas 63 está relacionada con disfunción plaquetaria y una mayor patogenicidad. ${ }^{[16]}$

La respuesta de citoquinas y quimiocinas inflamatorias aumenta, el TNF- $\alpha$ e INF- $\gamma$ causan cambios en la permeabilidad vascular $y$ son asociados a mayor severidad de la enfermedad. También hay aumento de IL-6 e IL-10, este último promueve la producción de anticuerpos, acrecentando la presencia de inmunocomplejos que se depositan en los tejidos además de que ejerce regulación hacia abajo de IL-12. ${ }^{[18]}$ Según Solà-Riera et al., las infecciones por HV también provocan que las células sean resistentes a la muerte mediada por receptores de ligandos inductores de apoptosis relacionados con TNF, ya que se ejerce una regulación hacia abajo sobre la expresión del antígeno HLA-DR5 a través de un mecanismo transitorio de degradación dependiente de proteasoma 26S, inhibiendo el transporte de este Rev Méd Cient. 2021;34(2): 16-23 antígeno hacia la superficie celular; por consiguiente, reprime la apoptosis mediada por linfocitos citotóxicos, sugiriendo que los HV pueden alterar el mecanismo de empalme de ARN en las células infectadas. ${ }^{[19]}$

\section{MANIFESTACIONES CLIINICAS}

Se describen cuatro fases clínicas: prodrómica, cardiopulmonar, diurética y de convalecencia (Ver Figura 3). La aparición de síntomas severos como insuficiencia respiratoria o choque es abrupta y puede ocurrir en las primeras 24 horas de hospitalización, siendo el periodo de mayor mortalidad. ${ }^{[6,20]}$

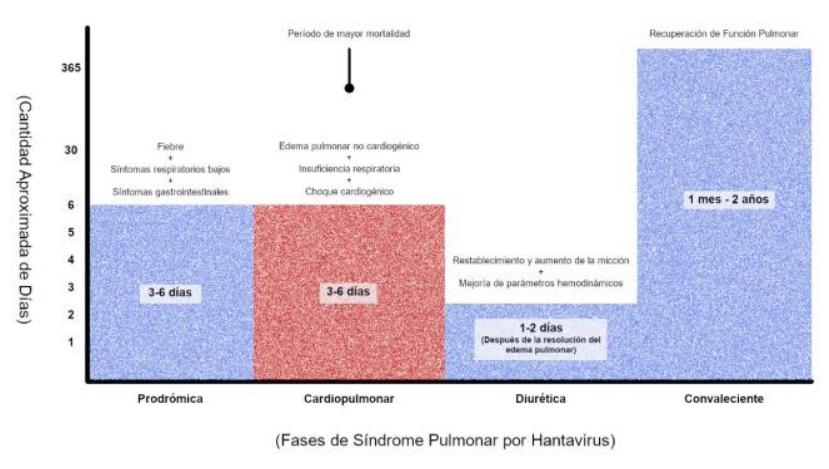

Figura 3. Fases clínicas del Síndrome Pulmonar por Hantavirus.

Fuente: Elaboración propia. Diseñada por Raúl GonzálezCastillo.

La fase prodrómica es la etapa inicial y febril, tiene una duración aproximada de 3-6 días, hay desarrollo de síntomas respiratorios bajos y síntomas gastrointestinales como náuseas, vómitos y dolor abdominal. ${ }^{[3,20]}$ La fase cardiopulmonar tiene una extensión de 3-6 días, se caracteriza por la aparición de edema pulmonar no cardiogénico asociado a disnea, anuria y tos no productiva, con evolución a choque cardiogénico. La fase diurética ocurre entre los 2-5 días después de la resolución del edema pulmonar, hay micciones aproximadas de 300 a 500 
$\mathrm{mL} / \mathrm{h}$, que acompañan la resolución rápida (24-48 horas) de las alteraciones a nivel respiratorio y hemodinámico. En la última fase de convalecencia, se presentan alteraciones residuales menores a nivel respiratorio (anomalías en la capacidad de difusión pulmonar), que puede comprender un periodo de meses a dos años hasta la recuperación completa. ${ }^{[3,21]}$

Los hallazgos de laboratorio no son específicos, en el conteo sanguíneo completo usualmente hay plaquetas menores de $150000 / \mathrm{mm}^{3}$, siendo esta trombocitopenia el hallazgo temprano más sensible que ocurre en la fase prodrómica y observándose hasta en un $80 \%$ de los pacientes; además, puede estar acompañado de leucocitosis con predominio de neutrófilos, diferencial con desviación de más de $50 \%$ hacia la izquierda, hematocrito elevado y presencia de linfocitos atípicos. El tiempo de protrombina puede estar prolongado y presentar leve aumento en creatinina, transaminasas, LDH, CPK, lactato sérico (>4.0 mmol/L), hipoalbuminemia y proteinuria. Todos los hallazgos mencionados están presentes desde la fase prodrómica, siendo más severos en la fase cardiopulmonar, y normalizándose en la fase convaleciente; sin embargo, con persistencia de la elevación del hematocrito. ${ }^{[21-22]}$

En la radiografía de tórax se suele encontrar en fases tempranas dos patrones: el primero con la presencia de un infiltrado intersticial difuso bilateral que evoluciona dentro de las primeras cuarenta y ocho horas a consolidación, efusión pleural y en dos tercios de estos pacientes se evidenciará gran afección del espacio aéreo perihiliar o bibasal, relacionados a una enfermedad agresiva y mortal. El segundo patrón es una imagen radiológica normal o con ligeros cambios que están ligados a la recuperación del paciente. Entre los hallazgos de tomografía de alta resolución, se pueden observar la presencia de opacidades de vidrio esmerilado, engrosamiento septal, efusión pleural, engrosamiento de la trama peribroncovascular y pequeños nódulos en más del $50 \%$ de los pacientes. ${ }^{[21,23]}$

\section{DIAGNÓSTICO}

Es importante considerar el SPH como diagnóstico diferencial en un escenario de insuficiencia respiratoria inexplicada y trombocitopenia. El uso de pruebas serológicas dentro de un contexto de sospecha clínica, es el método convencional para diagnosticar HV. Tradicionalmente, entre los 5-14 días después del comienzo de síntomas es el periodo comúnmente utilizado para realizar una prueba serológica; la técnica más empleada es el ELISA indirecto donde la presencia de IgM indica infección reciente, pero ocasionalmente también se puede encontrar IgG al inicio de los síntomas. ${ }^{[24]}$ La utilización de la inmunocromatografía para HV es relativamente reciente y aún en desarrollo. ${ }^{[25]} \mathrm{El}$ diagnóstico molecular mediante RT-PCR es efectivo y rápido, a pesar que se puede utilizar en tejido, tradicionalmente se emplea para detectar viremia y nos ayuda a identificar la especie de HV. Su uso tiene valores de sensibilidad de $93-94 \%$ y especificidad que alcanza el $100 \%$ en casos de HV del viejo y el nuevo mundo. ${ }^{[26]}$

TRATAMIENTO

No existe una terapia específica para el SPH, siendo el soporte vital un manejo primordial durante su hospitalización. ${ }^{[21]}$ Siempre se recomienda el monitoreo de estos pacientes en una unidad de cuidados intensivos. ${ }^{[3]}$ De presentar fracaso con un manejo convencional de cuidados críticos, la mejor opción para la sobrevida de los pacientes es la oxigenación por membrana extracorpórea (ECMO), teniendo un índice cardíaco menor de $2.3 \mathrm{~L} / \mathrm{min} / \mathrm{m}^{2}$, así como una relación de presión arterial de oxígeno 
y de fracción inspirada de oxígeno $\left(\mathrm{PaO}_{2} / \mathrm{FiO}_{2}\right)$ menor de 50. ${ }^{[21]}$ En el 2011, se publicaron resultados de la experiencia con el uso de ECMO, demostrando valores de hasta un $66.6 \%$ en sobrevida. ${ }^{[22]}$

El uso de glucocorticoides en infecciones por HV se extiende desde la Guerra de Corea en 1950, donde se administró cortisona intramuscular, disminuyendo la letalidad. La dexametasona se ha empleado en modelos animales y ha sido utilizada en conjunto con la metilprednisolona, para tratar la enfermedad en humanos. ${ }^{[27]}$ En el 2013, se realizó un ensayo clínico controlado, aleatorizado, doble ciego, cuyos resultados concluyeron que no existe beneficio clínico en el tratamiento de SPH con el uso de metilprednisolona intravenosa (IV) en altas dosis. ${ }^{[28]}$

Acerca de la ribavirina, al administrar una dosis de 50 $\mathrm{mg} / \mathrm{kg}$ IV, ha demostrado eficacia para disminuir la severidad de la insuficiencia renal y el requerimiento de diálisis cuando se utiliza en el curso temprano de la enfermedad con presentación SRHH, pero aún no se ha demostrado beneficio clínico para SPH. ${ }^{[21]}$ Tampoco se ha evidenciado la eficacia del uso de inmunoterapia con anticuerpos monoclonales y policlonales en las dos expresiones clínicas de la infección por HV en humanos. ${ }^{[27]}$ Se sugiere que la infusión de plasma humano convaleciente por el virus Andes es segura en el tratamiento de SPH; no obstante, más estudios son necesarios para su confirmación. ${ }^{[29]}$

Actualmente, dos vacunas han sido desarrolladas: una inactivada bivalente para el HTNV con un esquema de tres dosis, demostrando títulos de anticuerpos neutralizantes 33 meses tras el refuerzo en el $90 \%$ de los voluntarios; y la vacuna comercializada como Hantavax ${ }^{\mathrm{TM}}$ para el virus Seoul, obteniendo resultados de seroconversión del $97 \%$ a los 30 días seguidos del refuerzo y al año se evidenció que solo el $50 \%$ de los voluntarios tenían aún Rev Méd Cient. 2021;34(2):págs 16-23 anticuerpos neutralizantes. Se demostró reducción de la incidencia del SRHH, pero no de su severidad. Estas dos vacunas son utilizadas en China y Corea del Sur; sin embargo, para las cepas del nuevo mundo aún están en desarrollo. ${ }^{[27,30]}$

\section{AGRADECIMIENTOS}

Agradecemos al Dr. Samuel H. González V., especialista en Medicina Interna, por su asesoría y motivación brindada.

CONFLICTO DE INTERÉS

Los autores declaran no tener conflicto de interés.

BIBLIOGRAFÍA

[1] Abudurexiti A, Adkins S, Alioto D, Alkhovsky SV, AvšičŽupanc T, Ballinger MJ, et al. Taxonomy of the order Bunyavirales: update 2019. Arch Virol [Internet]. 2019 [citado 5 de abril de 2021]; 164(7):1949-65. Disponible en: https://link.springer.com/article/10.1007\%2Fs00705-01904253-6

[2] Schmaljohn CS, Dalrymple JM. Analysis of hantaan virus RNA: Evidence for a new genus of bunyaviridae. Virology [Internet]. 1983 [citado 15 de enero 2021]; 131(2):482-91. Disponible en: https://www.sciencedirect.com/science/article/abs/pii/00 42682283905147?via\%3Dihub

[3] Avšič-Županc T, Saksida A, Korva M. Hantavirus infections. Clin Microbiol Infect [Internet]. 2015 [citado 15 de enero 2021]; 21:6-16. Disponible en: https://www.clinicalmicrobiologyandinfection.com/article /S1198-743X(15)00536-4/fulltext

[4] Johnson N. The Role of Animals in Emerging Viral Diseases. Primera Edición. Editorial Academic Press; 2013. pp 235292. Disponible

en: https://linkinghub.elsevier.com/retrieve/pii/B9780124051 911000107

[5] Guzmán C, Mattar S, Calderón A. Diversity of rodents, hantavirus and its relationship to public health. Salud Uninorte [Internet]. 2015 [citado 15 de enero de 2021]; 31(3):584-98. Disponible en: http://www.scielo.org.co/scielo.php?script=sci arttext\&pi $\mathrm{d}=$ S0120-55522015000300015\&lang=es 
[6] Calderón GE, Brignone J, Martin ML, Calleri F, Sen C, Casas $\mathrm{N}$, et al. Brote de síndrome pulmonar por hantavirus, Tucumán, Argentina. Medicina (B Aires) [Internet]. 2018 [citado 15 de enero de 2021]; 78(3):151-157. Disponible en:

http://www.medicinabuenosaires.com/PMID/29940540.p df

[7] Vadell MV, Gómez Villafañe IE, Carbajo AE. Hantavirus infection and biodiversity in the Americas. Oecologia [Internet]. 2019 [citado 15 de enero de 2021]; 192(1):169$177 . \quad$ Disponible en: https://link.springer.com/article/10.1007\%2Fs00442-01904564-0

[8] Vollmar P, Lubnow M, Simon M, Müller T, Bergler T, Alois P, Thoma BR, Essbauer S. Hantavirus cardiopulmonary syndrome due to Puumala virus in Germany. J Clin Virol [Internet]. 2016 [citado 15 de enero de 2021]; 84:42-47. Disponible

en: https://www.sciencedirect.com/science/article/abs/pii/S1 386653216305492?via\%3Dihub

[9] Padula PJ, Edelstein A, Miguel SD, López NM, Rossi CM, Rabinovich RD. Hantavirus pulmonary syndrome outbreak in Argentina: molecular evidence for person-to-person transmission of Andes virus. Virology [Internet]. 1998 [citado 15 de enero de 2021]; 241(2):323-30. Disponible en:

https://www.sciencedirect.com/science/article/abs/pii/S1 386653216305492?via\%3Dihub

[10] Contreras-Matamala E, Luengo-Martínez C. Factores ambientales y síndrome cardiopulmonar por virus hanta en Chile. Rev salud pública [Internet]. 2020 [citado 15 de enero de 2021]; 22(1):1-7. Disponible en: http://www.scielo.org.co/scielo.php?script=sci_arttext\&pi $\mathrm{d}=\mathrm{S} 0124-00642020000100301$ \&lang=es

[11] Haines A, Patz JA. Health effects of climate change. JAMA [Internet]. 2004 [citado 22 de enero de 2021]; 291(1):99103. Disponible en: https://jamanetwork.com/journals/jama/articleabstract/197911

[12] Schmaljohn C, Nichol ST. Hantaviruses. Primera Edición. Editorial Springer; 2001. pp 47-75. Disponible en: https://link.springer.com/book/10.1007/978-3-64256753-7

[13] Klempa B, Radosa L, Kruger DH. The broad spectrum of hantaviruses and their hosts in Central Europe. Acta Virol [Internet]. 2013 [citado 15 de enero de 2021]; 57(2):130-7. Disponible en: http://www.elis.sk/index.php?page=shop.product details \&flypage=flypage.tp|\&product id=3419\&category id=107 \&option=com virtuemart\&vmcchk=1\&Itemid=1

[14] Romaní F, García Mendoza MP, Alarcón Villaverde JO. Frecuencia de anticuerpos contra Hantavirus en agricultores de arroz de una región tropical en el noreste del Perú. An Fac med [Internet]. 2020 [citado 1 de enero de 2021]; 81(1). Disponible en: https://revistasinvestigacion.unmsm.edu.pe/index.php/an ales/article/view/17155

[15] Vergote $V$, Laenen $L$, Vanmechelen $B$, Van Ranst $M$, Verbeken E, Hooper JW, Maes P. A lethal disease model for New World hantaviruses using immunosuppressed Syrian hamsters. PLoS NegI Trop Dis [Internet]. 2017 [citado 16 de enero de 2021]; 11(10):e0006042. Disponible en: https://journals.plos.org/plosntds/article?id=10.1371/jour nal.pntd.0006042

[16] Gavrilovskaya IN, Gorbunova EE, Mackow ER. Pathogenic hantaviruses direct the adherence of quiescent platelets to infected endothelial cells. J Virol [Internet]. 2010 [citado 16 de enero de 2021]; 84(9):4832-9. Disponible en: https://journals.asm.org/doi/10.1128/JVI.0240509?url ver $=$ Z39.88-

2003\&rfr id=ori:rid:crossref.org\&rfr dat=cr pub\%20\%20 Opubmed

[17] Schönrich G, Rang A, Lütteke N, Raftery MJ, Charbonnel N, Ulrich RG. Hantavirus-induced immunity in rodent reservoirs and humans. Immunol Rev [Internet]. 2008 [citado 22 de enero de 2021]; 225(1):163-89. Disponible en:

https://onlinelibrary.wiley.com/doi/abs/10.1111/j.1600065X.2008.00694.x

[18] Saksida A, Wraber B, Avšič-Županc T. Serum levels of inflammatory and regulatory cytokines in patients with hemorrhagic fever with renal syndrome. BMC Infect Dis [Internet]. 2011 [citado 22 de enero de 2021]; 11:142. Disponible

en: https://bmcinfectdis.biomedcentral.com/articles/10.1186 Ц1471-2334-11-142

[19] Solà-Riera C, Gupta S, Maleki KT, González-Rodriguez P, Saidi D, Zimmer CL, et al. Hantavirus Inhibits TRAILMediated Killing of Infected Cells by Downregulating Death Receptor 5. Cell Rep [Internet]. 2019 [citado 18 de enero de 2021]; 28(8):2124-2139.e6. Disponible en: https://www.cell.com/cell-reports/fulltext/S22111247(19)30970-

2? returnURL=https\%3A\%2F\%2Flinkinghub.elsevier.com\% 2Fretrieve\%2Fpii\%2FS2211124719309702\%3Fshowall\%3D $\underline{\text { true }}$ 
[20] Knust B, MacNeil A, Rollin PE. Hantavirus Pulmonary Syndrome Clinical Findings: Evaluating a Surveillance Case Definition. Vector Borne Zoonotic Dis [Internet]. 2012 [citado 19 enero de 2021]; 12(5):393-9. Disponible en: https://www.liebertpub.com/doi/10.1089/vbz.2011.0764 ?url ver=Z39.88-

2003\&rfr id=ori\%3Arid\%3Acrossref.org\&rfr dat=cr pub+ +0pubmed

[21] Hartline J, Mierek C, Knutson T, Kang C. Hantavirus infection in North America: a clinical review. AM J EMERG MED [Internet]. 2013 [citado 22 enero de 2021]; 31(6):978$82 . \quad$ Disponible en: https://www.sciencedirect.com/science/article/abs/pii/s0 735675713000703?via\%3Dihub

[22] Barclay CM. Síndrome cardiopulmonar por Hantavirus luego del brote de Epuyén, Argentina: la necesidad de investigar nuevas formas de intervención terapéutica. Acta Bioquím Clín Latinoam [Internet]. 2019 [citado 22 de enero 2021]; 245-251 Disponible en: https://www.redalyc.org/jatsRepo/535/53560335010/ht $\underline{\mathrm{ml} / \text { index.html }}$

[23] Barbosa D de L, Hochhegger B, Souza Jr. AS, Zanetti G, Escuissato DL, Meirelles $G$ de SP, et al. High-resolution computed tomography findings in eight patients with hantavirus pulmonary syndrome. Radiol Bras [Internet]. 2017 [citado 17 de enero 2021]; 50(3):148-53. Disponible en:

https://www.scielo.br/j/rb/a/XS69vbLSfpk4JVRWD5wYSV D/?lang=en

[24] Jonsson CB, Figueiredo LT, Vapalahti O. A global perspective on hantavirus ecology, epidemiology, and disease. Clin Microbiol Rev [Internet]. 2010 [citado 16 de enero de 2021]; 23(2):412-41. Disponible en: https://journals.asm.org/doi/10.1128/CMR.00062-09

[25] Amada T, Yoshimatsu K, Koma T, Shimizu K, Gamage CD, Shiokawa K, Nishio S, Ahlm C, Arikawa J. Development of an immunochromatography strip test based on truncated nucleocapsid antigens of three representative hantaviruses. Virol J [Internet]. 2014 [citado 22 de enero de 2021]; 11:87. Disponible en: https://virologyj.biomedcentral.com/articles/10.1186/174 3-422X-11-87

[26] Vial C, Martinez-Valdebenito C, Rios S, Martinez J, Vial PA, Ferres M, Rivera JC, Perez R, Valdivieso F. Molecular method for the detection of Andes hantavirus infection: validation for clinical diagnostics. Diagn Microbiol Infect Dis [Internet]. 2016 [citado 16 de enero de 2021]; 84(1):36-39. Disponible

en:
https://www.sciencedirect.com/science/article/pii/S0732 889315002813?via\%3Dihub

[27] Brocato RL, Hooper JW. Progress on the Prevention and Treatment of Hantavirus Disease. Viruses [Internet]. 2019 [citado 17 de enero de 2021]; 11(7):610. Disponible en: https://www.mdpi.com/1999-4915/11/7/610

[28] Vial PA, Valdivieso F, Ferres M, Riquelme R, Rioseco ML, Calvo $\mathrm{M}$, et al. High-Dose Intravenous Methylprednisolone for Hantavirus Cardiopulmonary Syndrome in Chile: A Double-Blind, Randomized Controlled Clinical Trial. Clin Infect Dis [Internet]. 2013 [citado 6 de enero 2021]; 57(7):943-51.

Disponible: https://academic.oup.com/cid/article/57/7/943/335822

[29] Vial PA, Valdivieso F, Calvo M, Rioseco ML, Riquelme R, et al. A non-randomized multicentre trial of human immune plasma for treatment of hantavirus cardiopulmonary syndrome by ANDV. Antivir Ther [Internet]. 2014 [citado 12 de enero de 2021]; 20(4):377-86. Disponible: https://www.intmedpress.com/journals/avt/abstract.cfm ?id=2875\&pid=48

[30] Yi Y, Park H, Jung J. Effectiveness of inactivated hantavirus vaccine on the disease severity of hemorrhagic fever with renal syndrome. Kidney Res Clin Pract [Internet]. 2018 [citado 8 de enero de 2021]; 37(4):366-72. Disponible en: https://www.krcpksn.org/journal/view.php?doi=10.23876/j.krcp.18.0044 